\title{
Latent tuberculosis infection in Mexican children with rheumatic diseases
}

\author{
Arturo Plascencia ${ }^{1}$, Iván Hernández ${ }^{2}$, Sergio Gutiérrez ${ }^{3}$, Antonio Luévanos ${ }^{1}$, César. E Juárez ${ }^{1}$, Manuel Sandoval ${ }^{5}$, Héctor R Pérez $^{4}$, Marcela \\ Plascencia $^{1}$, Edilberto González-Ochoa ${ }^{6}$ and Yaxsier de Armas ${ }^{7 *}$ \\ ${ }^{1}$ Servicio de Infectología Pediátrica, Hospital Civil de Guadalajara "Fray Antonio Alcalde”, México \\ ${ }^{2}$ Centro Universitario de Ciencia de la Salud, Universidad de Guadalajara, México \\ ${ }^{3}$ Servicio de Reumatología, Hospital Civil de Guadalajara "Fray Antonio Alcalde", México \\ ${ }^{4}$ Servicio de Infectología, Hospital Civil de Guadalajara "Fray Antonio Alcalde”, México \\ ${ }^{5}$ Secretaria de Salud, Jalisco, México \\ ${ }^{6}$ Grupo de Vigilancia e investigaciones en Tuberculosis y Lepra. Instituto de Medicina Tropical "Pedro Kourí", Cuba \\ ${ }^{7}$ Laboratorio de Biología Molecular del Hospital del Instituto de Medicina Tropical "Pedro Kouri”, Cuba
}

\begin{abstract}
Rheumatic diseases $(\mathrm{RD})$ patients have fourfold increased risk for tuberculosis infection compared with the general population. The aim of this work is to determine the prevalence of Latent Tuberculosis Infection (LTBI) in Mexican children and adolescents with RD. Descriptive exploratory study on 29 patients admitted in the Infectology Pedriatic Service at Civil Hospital "Fray Antonio Alcaide", Guadalajara, Jalisco, México from April 2013 - July 2014 was development. Patients were assessed for clinical and epidemiological data concerning LTBI and Tuberculosis Skin Test (TST) and Interferon Gamma Release Assay (IGRA, QuantiFERON tuberculosis Gold In-Tube (QFT-GIT)) results were compared. Prevalence of LTBI were 24.1 (TST) and 34.5\% (QFT-GIT), respectively; concordance was fair $(\mathrm{Kappa}=0.56(95 \% \mathrm{CI} ; 0.91-0.22))$. As the first study describing serial QFT-GIT and TST results from Mexican children, with RD, it reveals that they can be a reservoir of LTBI.
\end{abstract}

Tuberculosis (TB) is a global public health problem and a leading cause of morbidity and mortality worldwide. Children are a substantial part of the TB epidemic and it is estimated that there were 550,000 childhood TB cases globally ( $6 \%$ of total) in 2015 [1].

According to information provided by the Unique Platform of Information/SUIVE/DGE/SS DGISES/CUBESES 2015 in Mexico, during 2014, 21889 cases of TB (all forms) were registered, of them 20 137 new cases; $8.2 \%$ of the cases occurred in pediatric ages ( $\leq 18$ years), $21.2 \%$ associated to Diabetes Mellitus (DM) and 5.7\% to HIV infection. In the Jalisco state 1109 cases of TB (all forms) were notified, 1031 of them were new cases, being $11.4 \%$ pediatric cases, $17.5 \%$ and $6.8 \%$ were associated to DM and HIV, respectively [2].

Latent Tuberculosis Infection (LTBI) is the state in which human are infected with Mycobacterium tuberculosis without any clinical symptoms, radiological abnormality, or microbiological evidence. LTBI diagnosis is based on the targeted tuberculin skin test (TST) strategy, which recommends a test for the subjects who have risk factors for developing active TB. TST is used routinely in hospital clinical practice to screen for LTBI [3].

Recently, the Interferon Gamma Release Assay (IGRA), a T-cell based assay using $M$. tuberculosis specific antigens, has been increasingly used and studied for the diagnosis of LTBI. IGRA offers a more accurate approach than TST for the identification of individuals who have LTBI and might improve tuberculosis control by allowing for more precise targeting of preventive treatment [4].

Patients with Rheumatic Diseases (RD) have a fourfold increased risk of $\mathrm{TB}$ infection compared with the general population [5] However, in children and adolescents with $\mathrm{RD}$, data are still scarce and questions about the best strategy for LTBI screening and the risk of TB remain unanswered. In this study, we determined the prevalence of LTBI in Mexicans children and adolescents with RD comparing IGRA and TST result.

We conducted a descriptive exploratory study comparing IGRA and TST in 29 cases of RD admitted in the Infectology Pedriatic Service at Civil Hospital "Fray Antonio Alcalde", Guadalajara, Jalisco, México from April 2013 to July 2014. Verbal and written informed consent was obtained from the parents or guardians on behalf of the children enrolled in the study.

The TST was performed with an intradermic injection of 2 tuberculin units (TU) of PPD TUBERSOL ${ }^{\circledR}$ (Sanofi Pasteur Ltd, Canada) and read 72 hours thereafter. A strong TST reaction was defined when induration was $\geq 10 \mathrm{~mm}$ according to the protocols of the World Health Organization (WHO) [6].

IGRA Assay (QFT-GIT) by means of QuantiFERON tuberculosis

Correspondence to: Y. de Armas, Laboratorio de Biología Molecular del Hospital del Instituto de Medicina Tropical "Pedro Kourí", Cuba, Tel: 537 2553257; E-mail: Yaxsier@ipk.sld.cu

Key words: latent tuberculosis, rheumatic diseases, children, quantiferon, tuberculosis skin test

Received: July 02, 2016; Accepted: August 01, 2016; Published: August 04, 2016 
Gold In-Tube [QFT-GIT] (Cellestis Ltd, Victoria, Australia) tests were performed according to the manufacturer's instructions. Briefly, whole blood was collected by venipuncture from each subject and incubated for 16-24 hours in 3 separate conditions: 1) a mixture of 3 TB antigens from ESAT-6, CFP-10, and TB7.7; 2) a mitogen as a positive control; and 3) a negative control (nil). Following the stimulations, $150 \mu \mathrm{L}$ of the supernatant was harvested from each tube. Then, $50 \mu \mathrm{L}$ of each supernatant was used to determine its interferon gamma concentration by the ELISA. A QuantiFERON value of 0.35 international units or more was deemed positive according to manufacturer's instructions.

Statistical analysis: Frequencies and proportions were used to present the categorical data. Median and standar deviation were calculates for numerical data.

The agreement between QFT-Gold test and TST was calculated using Cohen's Kappa coefficient. K values greater than 0.75 , between 0.4 and 0.75 , and less than 0.4 were considered excellent, fair and poor agreement, respectively [6]. The research protocol was revised and approved by Hospital Civil de Guadalajara "Fray Antonio Alcalde": 086/12 and Secretaria de Salud Jalisco:15/HCGFA-JAL/2013.

Out of the total 16838 patient attending the Hospital's external consultation, 126 were remitted to Rheumatology Service due to suspicion of $\mathrm{RD}$; in 29 of them RD were confirmed and screened for LTBI. Result of TST and (QFT-GIT) with demographic and clinic data of RD cases is given in Table 1 .
The median age and standard deviation of the participants were 12.10 (SD 3.95 years), 20 were female patients (69.0\%). Eleven patients (37.9\%) were juvenile idiopathic arthritis (JIA), seven (24.1\%) systemic lupus erythematous (SLE) and two (6.9\%) with concomitant diseases. 93.1\% of the children enrolled in this study received BCG vaccine at birth. Four cases had positive epidemiological history for TB and only one developed LTBI. The median of treatment duration and standard deviation were 330 days (SD 397).

Prevalence of LTBI were 24.1 (TST) and 34.5\% (QFT-GIT) respectively. Concordance between tests was fair (Kappa $=0.56(95 \%$ CI; 0.91-0.22)).

This is, to our best knowledge, the first study that describes serial QFT-GIT and TST results from Mexican children with different RD. The prevalence of LTBI were 24.1 (TST) and 34.5\% (QFT-GIT), respectively. Apparently is comparable with the estimation that onethird of the world's population is infected with M. tuberculosis [1].

Different reports are suggesting an increased incidence of tuberculosis infection in children with chronic rheumatic disease $[3,5]$. Many RD patients have long-term treatment with corticosteroids and with Biologic Response Modifiers (BRMs) that increase their risk of LTBI reactivation. Here, all patients' received treatment for long duration. Several studies have associated corticosteroid use with reactivation risk. Also antitumor necrosis factor- $\alpha$ (TNF- $\alpha$ ) therapy is strongly associated with reactivation of TB in patients with $\mathrm{RD}$. TB

Table 1. Result of TST and QFT-GIT with the demographic and clinic profile of RD cases enrolled in this study ( $\mathrm{n}=29$ ).

\begin{tabular}{|c|c|c|c|c|c|c|c|c|}
\hline No & $\begin{array}{c}\text { Age } \\
\text { (years) }\end{array}$ & Gender & BCG vaccine & $\begin{array}{c}\text { Contact } \\
\text { TB }\end{array}$ & TST & $\begin{array}{l}\text { QFT } \\
\text { GIT }\end{array}$ & RD & $\begin{array}{c}\text { Treatment duration } \\
\text { (days) }\end{array}$ \\
\hline 1 & 11 & $\mathrm{M}$ & + & - & - & - & JIA & 10 \\
\hline 2 & 11 & $\mathrm{~F}$ & + & - & - & - & JIA & 180 \\
\hline 3 & 15 & $\mathrm{~F}$ & + & - & - & - & SLE+ SS & 1460 \\
\hline 4 & 5 & $\mathrm{~F}$ & + & - & - & - & JIA & 1 \\
\hline 5 & 13 & M & + & - & - & - & MO & 6 \\
\hline 6 & 10 & $\mathrm{~F}$ & + & + & - & - & JIA & 90 \\
\hline 7 & 15 & M & + & - & - & - & SLE & 365 \\
\hline 8 & 13 & $\mathrm{~F}$ & + & - & - & + & ES & 90 \\
\hline 9 & 16 & M & + & - & - & - & JIA & 1825 \\
\hline 10 & 14 & M & + & - & + & + & JS & 730 \\
\hline 11 & 17 & $\mathrm{~F}$ & + & + & - & - & $\mathrm{D}$ & 730 \\
\hline 12 & 9 & M & + & - & + & + & JIA & 730 \\
\hline 13 & 17 & $\mathrm{~F}$ & + & - & + & + & AS & 180 \\
\hline 14 & 11 & M & + & - & - & - & JIA & 1460 \\
\hline 15 & 4 & $\mathrm{~F}$ & + & - & - & - & HSS & 90 \\
\hline 16 & 4 & $\mathrm{~F}$ & + & + & - & - & JIA & 730 \\
\hline 17 & 11 & $\mathrm{M}$ & + & - & - & - & SLE + AS & 21 \\
\hline 18 & 11 & $\mathrm{~F}$ & + & - & + & + & JIA & 1095 \\
\hline 19 & 15 & $\mathrm{~F}$ & + & - & - & + & JIA & 30 \\
\hline 20 & 5 & $\mathrm{~F}$ & + & - & - & - & $\mathrm{D}$ & 210 \\
\hline 21 & 13 & M & + & + & + & + & SLE & 240 \\
\hline 22 & 10 & $\mathrm{~F}$ & - & - & - & - & SLE & 14 \\
\hline 23 & 15 & M & + & - & - & - & LE & 730 \\
\hline 24 & 13 & $\mathrm{~F}$ & + & - & + & + & SLE & 270 \\
\hline 25 & 16 & M & + & - & + & + & JIA & 730 \\
\hline 26 & 14 & $\mathrm{M}$ & + & - & - & - & PP & 1095 \\
\hline 27 & 17 & $\mathrm{~F}$ & + & - & - & - & JIA & 2555 \\
\hline 28 & 9 & M & + & - & - & + & CSS & 120 \\
\hline 29 & 17 & $\mathrm{~F}$ & - & - & - & - & SLE & 540 \\
\hline
\end{tabular}

Legend: M: Male; F: Female +: Positive Result: -: Negative Result; JIA: Juvenile Idiopathic Arthritis;SLE: Systemic Lupus Erytematosus; SS: Sjögren Syndrome; MO: Myelitis Optical; ES: Evans Syndrome; JS: Juvenile Spondyloarthropathy; D: Dermatomyositis; AS: Antiphospholipid Syndrome; HHS: Henoch Shleiden Syndrome; LE: Localized Scleroderma; PP: Pars Planitis; CSS: Churg Straus Syndrome. 
screening before starting BRMs reduces the incidence of reactivation of LTBI infection by up to $85 \%[7,8]$.

Diagnosis of TB in children is often challenging. One limitation to the diagnosis of LTBI is the lack of a "gold standard" test. TST has been a diagnostic tool for determining previous infection with M. tuberculosis. However, the sensitivity of TST is still considerably lower, especially in immunocompromised subjects. TST contains more than 200 proteins widely shared among micobacteria other than M. tuberculosis, including M. bovis, BCG and many non-tuberculous micobacteria (NTM) [6].

Recently, two commercially test the QuantiFERON-TB In-Tube (enzyme linked immunosorbent assay) and T. SPOT.TB $\%$ ELISPOT (enzyme linked immunospot assay) are available to detect LTBI. In general, IGRA correlates better than TST with exposure to infectious patients and shows higher sensitivity to active TB than the TST [4]. However, previous studies, indeterminate QFT-GIT results accounted for $21.4 \%$ of all patients with immunosuppression. Observed discrepancies may be related to several technical and interpretation aspects related to test methodology. Recent data from UK clinical research verified that the LTBI detection rate was markedly increased when 3 methods (clinical factors, TST, and IGRA) were used in combination [9].

In this study, QFT-GIT and TST had fair levels of agreement. Results of fifteen studies (included in meta-analysis) showed that there were no significant differences in sensitivity between TST $(88.2 \%$, 95\% confidence interval [CI] 79.4- 94.2\%) and QFT-IT (89.6\%, 95\% CI 79.7-95.7\%) in immunocompetent children [10]. However, in children and adolescents with RD, data are still scarce and some studies are awarded. On the other hand, Pérez-Porcuna and co-worker conferred the discordant results (TST vs QFT-GIT) to the presence of intestinal helminths, the length of exposure to an index case and blood ferritin levels. However, those aspects were not evaluated in our study. Although both tests are valuable in screening for LTBI, the diagnostic accuracy varies according to the patient population [11].

Two recent studies reported the incidence of TB in close contacts to be as high as $77 \%-89 \%[12,13]$. Surprisingly, here only one contact developed LTBI. The lower incidence in our series might be due to the small cases analyzed. Other possible explanation is the different immune response in relation to the number of infection observed.

So far, studies in children are required to assess the effect of factors such as malnutrition, non-tuberculosis mycobacterial infections, HIV and parasitic infections on the performance of the test. Minimizing the risk of progression of LTBI to active TB is necessary.

\section{Transparency declaration}

The authors declare no conflicts of interest.

\section{References}

1. World Health Organization. Global Tuberculosis Control: WHO report 2015. Geneva: World Health Organization, Geneva, Switzerland, 2015.

2. Dirección General de Epidemiologia, Secretaria de Salud, México 2016.

3. Sztajnbok F, Boechat NL, Ribeiro SB, Oliveira SK, Sztajnbok DC, et al. (2014) Tuberculin skin test and ELISPOT/T. SPOT.TB in children and adolescents with juvenile idiopathic arthritis. Pediatr Rheumatol Online J 12: 17. [Crossref]

4. Song SE, Yang J, Lee KS, Kim H, Kim YM, et al. (2014) Comparison of the tuberculin skin test and interferon gamma release assay for the screening of tuberculosis in adolescents in close contact with tuberculosis TB patients. PLoS One 9: e100267. [Crossref]

5. Agarwal S, Kumar Das S, Agarwal GG, Srivastava R (2014) Steroids decrease prevalence of positive tuberculin skin test in rheumatoid arthritis: implications on antiTNF therapies. Interdiscip Perspect Infect Dis.

6. Stop TB Partnership Childhood TB Subgroup World Health Organization (2006) Guidance for National Tuberculosis Programmes on the management of tuberculosis in children. Chapter 1: introduction and diagnosis of tuberculosis in children. Int J Tuberc Lung Dis 10: 1091-1097. [Crossref]

7. Mehta B, Zapantis E, Petryna O, Efthimiou P (2015) Screening Optimization of Latent Tuberculosis Infection in Rheumatoid Arthritis Patients. Arthritis 2015: 569620. [Crossref]

8. Chen DY, Shen GH, Hsieh TY, Hsieh CW, Lan JL (2008) Effectiveness of the combination of a whole-blood interferon-gamma assay and the tuberculin skin test in detecting latent tuberculosis infection in rheumatoid arthritis patients receiving adalimumab therapy. Arthritis Rhem 59: 800-806. [Crossref]

9. Shim TS (2014) Diagnosis and Treatment of Latent Tuberculosis infection in patients with inflammatory bowel diseases due to initiation of anti-tumor necrosis factor therapy. Intestinal Res 12: 12-19.

10. Laurenti P, Raponi M, de Waure C, Marino M, Ricciardi W, et al. (2016) Performance of interferon-? release assays in the diagnosis of confirmed active tuberculosis in immunocompetent children: a new systematic review and meta-analysis. BMC Infect Dis 16:131.

11. Pérez-Porcuna TM, Ascaso C, Malheiro A, Abellana R, Martins M, et al. (2014) Mycobacterium tuberculosis infection in young children: analyzing the performance of the diagnostic tests. PLoS One 9: e97992. [Crossref]

12. Hanifa Y, Grant AD, Lewis J, Corbett EL, Fielding K, et al. (2009) Prevalence of latent tuberculosis infection among gold miners in South Africa. Int J Tuberc Lung Dis 13: 39-46. [Crossref]

13. Lawn SD, Wood R, De Cock KM, Kranzer K, Lewis JJ, et al. (2010) Antiretrovirals and isoniazid preventive therapy in the prevention of HIV-associated tuberculosis in settings with limited health-care resources. Lancet Infect Dis 10: 489-498. [Crossref]

Copyright: (C2016 Plascencia A. This is an open-access article distributed under the terms of the Creative Commons Attribution License, which permits unrestricted use, distribution, and reproduction in any medium, provided the original author and source are credited. 